\title{
Rapid Diagnostic Test Versus Microscopy for Diagnosing Malaria Among Pregnant Women in a Resource-Poor Setting; A Cross-Sectional Comparative Study
}

\author{
Bartholomew N. Odio ${ }^{1}$, Leonard O. Ajah ${ }^{1,2}$, Perpetus C. Ibekwe ${ }^{1}$, Monique I. Ajah ${ }^{3}$, George O. Ugwu ${ }^{2}$, \\ Theophilus O. Nwankwo ${ }^{2} \&$ Christian C. Anikwe ${ }^{1}$ \\ ${ }^{1}$ Department of Obstetrics and Gynaecology, Alex Ekwueme Federal University Teaching Hospital, Abakaliki, \\ Nigeria \\ ${ }^{2}$ Department of Obstetrics and Gynaecology, Faculty of Medical Sciences, University of Nigeria, Ituku-Ozalla \\ Campus, Enugu, Nigeria \\ ${ }^{3}$ Institute of Maternal and Childhealth, Faculty of Medical Sciences, University of Nigeria, Ituku-Ozalla Campus, \\ Enugu, Nigeria \\ Correspondence: Dr. Leonard Ogbonna Ajah, Department of Obstetrics and Gynaecology, Faculty of Medical \\ Sciences, University of Nigeria, Ituku-Ozalla Campus, Enugu, Nigeria. E-mail: leonard.ajah@unn.edu.ng
}

Received: April 13, 2020 Accepted: May 11, 2020 Online Published: May 29, 2020

doi:10.5539/gjhs.v12n8p52 URL: https://doi.org/10.5539/gjhs.v12n8p52

\begin{abstract}
Background: Diagnostic challenge of malaria in Nigeria remarkably impedes the World Health Organization (WHO) recommendation of laboratory diagnosis before treatment. Rapid Diagnostic Test (RDT) is easier and cheaper to perform when compared with microscopy especially in resource-poor settings. However there are conflicting results on the accuracy of RDT versus microscopy from previous studies.
\end{abstract}

Aim: To compare the overall accuracy of microscopy and RDT in detecting peripheral malaria among pregnant women with clinical features of malaria.

Materials and Methods: This was a cross-sectional comparative studyin whichRDT, microscopy and polymerase chain reaction (PCR) were performed using the peripheral bloodof the eligible study participants at the Alex Ekwueme Federal University Teaching Hospital, Abakaliki between September 1, 2016 and March 31, 2017.The PCR was used as the gold standard in this study. Data was analyzed with the Statistical Package for Social Sciences version 18 (IBM SPSS, Chicago, USA). P value $\leq 0.05$ was considered statistically significant.

Results: The actual prevalent rates of malaria based on RDT, microscopy and PCR results among the participants were $58.2 \%, 59.9 \%$ and $61.1 \%$ respectively. There was no statistical significant difference among RDT, microscopy and combined RDT and microscopy on overall accuracy. Malaria infestation was associated with self-employed and unemployed women, primigravidity, second trimester, rural residence, non-use of long lasting insecticide treated nets and intermittent preventive therapy for malaria.

Conclusion: There was no difference in overall accuracy among RDT, microscopy and combined RDT and microscopy. This underscores the need to scale up RDT for every patient with clinical features of malaria before treatment in this environment.

Keywords: malaria in pregnancy, rapid diagnostic test, microscopy, polymerase chain reaction, South-East Nigeria

\section{Introduction}

Malaria constitutes the most common cause of out-patient visits in Nigeria (FMH, 2015). Because of this, substantial resources are lost to the disease annually (FMH, 2014). The most vulnerable groups are under 5 children and gravid women (FMH, 2014). Among the pregnant women, malaria infects more in second trimester and, in extreme situations, may extend to the puerperium (FMH, 2015).Malaria endemicity in the tropics is worsened by the resistance of plasmodium parasite to the available anti-malarial drugs and lack of malaria vaccine (WHO, 2015). 
Conventional light microscopy of blood smear is sensitive and can detect densities as low as 100 parasites $/ \mu \mathrm{L}$ of blood (WHO, 1988). It diagnoses the parasite species, densities, circulating stages and helps in parasitological response to chemotherapy. However, the diagnostic challenge of conventional microscopy is that it is unable to detect all infections as parasites can be sequestered in the placenta and this increases the maternal and perinatal morbidity and mortality (Anchang-Kimbiet al., 2009; Mockenhauptet al., 2006; Adam et al., 2005; Leke et al., 1999). Microscopy is also labour-intensive and delays treatment. Such delays make the clinicians presumptively treat the patients before the results are released. This is against the recommendation of routine laboratory diagnosis of malaria before treatment (Perkins et al., 1999). Furthermore, microscopy depends on good techniques, facilities and well trained individuals (WHO, 1988). These conditions are hardly met at the resource-limited settings like Nigeria. The use of laboratory methods has become necessary because care givers cannot objectively identify malaria cases using clinical features alone (Perkins et al., 1999).

Rapid diagnostic tests (RDTs) detect either histidine rich protein-2 (HRP-2) or Plasmodium lactate dehydrogenase $(\mathrm{pLDH})$ produced by infected red blood cells. Although majority of RDTs detect antibodies against HRP-2 or pLDH, a significant proportion of tests include a Pan antialdolase test line. RDTs are recommended by the World Health Organisation(WHO) to enhance prompt diagnosis and treatment in order to prevent complications due to delayed treatment. It overcomes the drawbacks of defective microscope and unstable power supply especially in resource-poor settings like Nigeria. Health workers with minimal skills can be trained on RDT techniques within short period of time. According to WHO, RDTs must be able to detect 100 parasites $\mu \mathrm{l}^{-1}$ and have a high overall accuracy (Abba et al., 2011).

There is lack of trained manpower and tools for malaria microscopy in Nigeria especially in the rural areas hence most patients are treated presumptively. Malaria RDTs are simple, easy to use with little expertise required for interpretation and result is obtained within minutes. RDTs are very useful in endemic areas where many people can be screened in a short period of time. Based on med line search, there are very few studies that compared the overall accuracy of conventional microscopy with rapid diagnostic test for malaria among pregnant women using the polymerase chain reaction as the gold standard. Even these few previous studies produced conflicting results hence the need for this study. This study was therefore aimed at comparing the overall accuracy of conventional microscopy and RDTs in detecting peripheral malaria in pregnant women with clinical features of malaria. The outcome of this study may be used to make informed decisions on scaling up the use of RDTs or otherwise, especially in rural areas where manpower for microscopy are not readily available, thus enhancing the WHO policy on testing before treatment of malaria.

\section{Methods}

\subsection{Study Area}

Ebonyi state lies entirely in the Cross River plains with frequent floods during the rainy season, resulting from poor drainage systems, stagnant streams and ponds which predispose the state to mosquito infestations and malaria endemicity. The bulk of population in Ebonyi State are poor and most of them live in rural areas. The Alex Ekwueme Federal University Teaching Hospital, Abakaliki (formerly Federal Teaching Hospital, Abakaliki) is located in Abakaliki, the capital of Ebonyi state. The hospital serves the population within and around Abakaliki metropolis and also as a referral centre within the state. The hospital has a functional Obstetrics and Gynaecology Department.

\subsection{Study Design}

This was a cross sectional comparative study in which consenting antenatal clinic attendees with clinical features of malaria were recruited. The study was carried out between September 1, 2016 and March 31,2017. A semi-structured questionnaire was used to collate information from the eligible participants on the socio-demographic characteristics and the RDT, microscopy and PCR results.

\subsection{Laboratory Procedures}

Three milliliters(mls) of blood were collected from the ante-cubittal veins of the less dependent forearm of the study participants and put into two different ethylenediaminetetraacetic acid(EDTA) bottles in the ratio of 2:1 . The sample bottle containing $1 \mathrm{ml}$ of blood was used for RDT while the one containing $2 \mathrm{mls}$ of blood was used for microscopy and PCR.

\subsection{Rapid Diagnostic Testing for Malaria}

The RDT kit, SD Bioline, was used in this study. It was used for detection of the antigen-Histidine Rich Protein-2 (PfHRP2) and enzyme-lactate dehydrogenase (pfLDH). Each box of 25 individually sealed strips with a desiccant 
was supplied with loops, alcohol swabs and lancets. The WHO standard procedure (WHO 2010), was used for RDT tests in this study.

\subsection{Microscopy}

Thin and thick blood smears were made from $1 \mathrm{ml}$ of collected sample; stained with Giemsa and then examined under the microscope using x100 objective lens. Also the WHO standard procedure (WHO 2009), was used for malaria microscopy. The 2 microscopists who viewed the slides independent of each other were WHO certified. A consultant microbiologist viewed the slide where there was discordant results from the 2 microscopists.

\subsection{Polymerase Chain Reaction (PCR)}

The remaining $1 \mathrm{ml}$ of blood sample was subjected to molecular assessment. The standard procedure (Tan et al., 1997), was used for PCR in this study.

The consenting pregnant women with features of malaria from the $14^{\text {th }}$ week gestation (second trimester) were included for this study. However the pregnant women without features of malaria, those with features of malaria in the first trimester and those who declined consent despite adequate counseling, were excluded from the study.

\subsection{Sample Size Determination}

The sample size was calculated with the formula for cross sectional studies (Charan \& Biswas, 2013);

$\mathrm{N}=\mathrm{Z}_{\alpha / 2}{ }^{2} \mathrm{P}(1-\mathrm{P}) / \mathrm{d}^{2}$. Where $\mathrm{N}=$ Sample size; $\mathrm{Z}_{\alpha / 2}=$ Standard normal variate at $95 \%$ confidence interval and power of $80 \%=1.96 ; \mathrm{P}=$ prevalence of malaria parasitaemia in pregnancy. From a previous study carried out in Abakaliki, the prevalence of malaria parasitaemia in pregnancy was $29 \%$ (Nwonwu et al., 2009); $d=$ desired precision at $95 \%$ confidence interval $=0.05$. Adding $10 \%$ attrition rate, the sample size for this study was 348 .

\subsection{Data Collection and Analysis}

Data was analyzed with the Statistical Package for Social Sciences version 18 (IBM SPSS, Chicago, USA). Results were categorized as positive and negative for both RDTs and microscopy. Sensitivity, specificity Positive Predictive Value (PPV), Negative Predictive Value (NPV) and overall accuracy were calculated by comparing the proportion of positive and negative results for RDT and microscopy. Categorical variables were compared using chisquare test where applicable. P value $\leq 0.05$ was considered statistically significant.

\subsection{Ethics Approval and Informed Consent}

Ethical approval for this study was obtained from the Research Ethics Committee of Alex Ekwueme Federal University Teaching Hospital, Abakaliki. The ethical clearance registration number and date were FETHA/REC/VOL 1/2016/364 and 18th May,2016 respectively. Informed consent was obtained from the eligible participants before they were enrolled for the study.

\section{Results}

Out of 350 eligible pregnant women recruited for this study, it was only 347 of them with complete data that was analyzed. Table 1 shows the socio-demographic characteristics of the respondents. Majority of the respondents were between 25 and 34 years, had at least secondary education and $\leq 3$ gravidity and were in their second trimester. Table 2 shows the correlation between RDT and microscopy with PCR results. The prevalence of malaria using the RDT, microscopy and PCR among the respondents were $58.2 \%, 59.9 \%$ and $61.1 \%$ respectively. The tests of validity of the methods is shown in table 3 . The overall accuracy of RDT, microscopy and combined RDT and microscopy were $83.9 \%, 82.1 \%$ and $85.6 \%$ respectively.

The comparison of the overall accuracy of the test methods is shown in Table 4. There was no statistical significant difference between RDT and microscopy as well as combined RDT and microscopy and the individual methods on overall accuracy ( $\mathrm{P}$-value $=>0.05$ ). Table 5 shows theassociation of respondents' socio-demographic characteristics with PCR test outcome. Malaria infestation was significantly more common among self-employed and unemployed pregnant women, primigravid women, women in second trimester of pregnancy, non-users of long lasting insecticide treated nets (LLITN),rural dwellers and those who did not receive intermittent preventive therapy for malaria. Figure 1 shows the symptoms at presentation. Majority of the participants had fever.

\section{Discussion}

This study showed that the actual prevalence of malaria based on RDT, microscopy and PCR results among pregnant women with clinical features of malaria were $58.2 \%, 59.9 \%$ and $61.1 \%$ respectively. There was no statistical significant difference among RDT and microscopy, RDT and combined RDT and microscopy, as well as microscopy and combined RDT and microscopy on overall accuracy. Malaria infection was associated with 
self-employed and unemployed women, primigravidity, second trimester, rural residence and non-use of malaria preventive measures.

The $59.9 \%$ prevalence of malaria based on the microscopy results among pregnant women in this study is higher than $42 \%$ and $49.8 \%$ previously reported in Abakaliki, South-East Nigeria and Sagamu,South-West Nigeria respectively (Sule-Odu et al., 2016; Odikamnoro et al., 2014). The possible reason for this disparity could be attributed to the malaria investigation being performed on the pregnant women with clinical features of malaria in this study unlike the previous studies that were carried out on asymptomatic women. The prevalence of malaria in decreasing order of detection of PCR followed by microscopy and then RDT in this study is supported by a similar study in Jos, North-Cenral Nigeria (Ita et al., 2018). However this is in contrast with the report by Kanyi et al., in Lagos in which the decreasing order of malaria prevalence based on detection by PCR was followed by RDT and finally by microscopy (Kanyi et al., 2016). This study showing that $38.9 \%$ of participants with clinical features of malaria were not caused by malaria but by other pathogens is essentially similar to $41.1 \%$ reported in Cameroun (Mfuh et al., 2019). This underscores the need for confirmation of malaria before administration of anti-malarial drugs to avoid abuse of the drugs, reduce economic wastage and resistance of malarial parasites to anti-malarial drugs.

WHO recommends that RDT assays must achieve $90 \%$ specificity and $95 \%$ sensitivity to be universally used for the diagnosis of malaria (WHO 2018).However previous RDT evaluation surveys showed that many RDT kits achieved this target in non-pregnant women but difficult in pregnant women (WHO 2012). The pregnant women being the study participants in this study could have accounted for the lower sensitivity and specificity recorded when compared with the WHO target. The NPV of RDT being slightly higher than that of microscopy in this study suggests that a negative RDT result has a better re-assurance to the patient that the symptoms and signs are not due to malaria but other causes when compared with microscopy. The pfLDH disappears from the blood within 24-48 hours of malaria parasite clearance while the pfHRP2 can persist for weeks in the blood after malaria parasite clearance. The similarity between the pfLDH and pfHRP2 results in this study suggests that there are active malaria infections among the RDT positive respondents.

Malaria infection being significantly associated with the low parity ,rural dwelling, low income earners, and lack of use of preventive measuresin this study is supported by previous reports from South-west and north-west, Nigeria (Aliyu et al., 2017; Agomo \& Oyibo, 2013). Age not having a significant effect on malaria infection in this study is contrary to the report in Lagos, Nigeria (Agomo \& Oyibo, 2013). A significant proportion of the pregnant women who had malaria infestation in second trimester in this study is essentially similar to the previous report in Nigeria (FMH, 2014). However it differed from the report in Kaduna, North-west Nigeria (Aliyuet al., 2017).

This study was strengthened by the standard procedures applied during the RDT, microscopy and PCR. It was however weakened by some of the information sought for which were prone to recall bias. Despite the standard procedures applied during the RDT, microscopy and PCR, there could also be intra and inter-observer errors.

In conclusion, there was no difference in overall accuracy among RDT, microscopy and combined RDT and microscopy. This underscores the need to scale up RDT for every patient with clinical features of malaria before treatment in this environment. This will help reduce abuse of the available anti-malarial drugs and chemo-resistance to malarial parasites.

\section{Acknowledgements}

We wish to thank Ebonyi state malaria elimination program unit for providing all the malaria rapid diagnostic test kits that were used for this study and the authors for funding the PCR and microscopy that were performed on the study participants.

\section{Competing Interests Statement}

All authors have no conflicts of interest to declare.

\section{References}

Abba, K., Deeks, J. J., Olliaro, P., Naing, C. M., Jackson, S. M., \& Takwoingi, Y. (2011). Rapid diagnostic test for diagnosing uncomplicated P. falciparum malaria in endemic countries. Cochrane Database Systems Rev., 7, CD008122. https://doi.org/10.1002/14651858.CD008122.pub2

Adam, I. E., Salih, I., \& Elbashir, M. I. (2005). Submicroscopic Plasmodium falciparum infections during pregnancy, in an area of Sudan with a low intensity of malaria transmission. Ann Trop Med Parasitol., 99, 339-344. https://doi.org/10.1179/136485905X36244

Agomo, C. O., \& Oyibo, W. A. (2013). Factors associated with risk of malaria infection among pregnant women in 
Lagos, Nigeria. Infect Dis Poverty, 2(1), 19. https://doi.org/10.1186/2049-9957-2-19

Aliyu, M. M., Nasir, I. A., Umar, Y. A., Vanstawa, A. P.,Medugu, J. T.,Emeribe, A. U., et al. (2017). Prevalence, risk factors, and antimalarial resistance patterns of falciparum plasmodiasis among pregnant women in Kaduna metropolis, Nigeria. Ci Ji Yi Xue Za Zhi, 29(2), 98-103. https://doi.org/10.4103/tcmj.tcmj_22_17

Anchang-Kimbi, J. K., Achidi, E. A., Nkegoum, B., Sverremark-Ekstrom, E., \& Troye-Blomberg, M. (2009). Diagnostic Comparison of malaria infection in peripheral blood and placental biopsies in Cameroonian parturient women. Malar J., 8, 126. https://doi.org/10.1186/1475-2875-8-126

Charan, J., \& Biswas, T. (2013). How to Calculate Sample Size for Different Study Designs in Medical Research? Indian J Psychol Med., 35(2), 121-126. https://doi.org/10.4103/0253-7176.116232

Federal Ministry of Health. (2014). National Malaria Strategic Plan 2014- 2020. Abuja. National Malaria Elimination Programme, 2014.

Federal Ministry of Health. (2015). National Guidelines for Diagnosis and Treatment of Malaria. National Malaria and Vector Control Division Abuja-Nigeria, 3, 1-61.

Ita, I. O., Out, A. O.,Onyedibe, K., Iwuafor, A. A., Banwat, E., \& Egah, D. E. (2018). A diagnostic performance evaluation of rapid diagnostic tests and microscopy for malaria diagnosis using nested polymerase chain reaction as reference standard in a tertiary hospital in Jos, Nigeria. Transactions of The Royal Society of Tropical Medicine and Hygiene, 112(10), 436-442. https://doi.org/10.1093/trstmh/try071

Kanyi, O. I., Ajayi, M. B., Ezeugwu, S. M. C., Afocha, E. E., \& Iwalokun, B. (2016). Comparison of Rapid Diagnostic Test (RDT), Polymerase Chain Reaction (PCR) and microscopy methods in the diagnosis of malaria among airport workers in Lagos. Nigerian Journal of Science and Environment, 13(1), 18-24.

Leke, R. G., Djokam, R., Mbu, R., Leke, F. J., Fogako, J., Megnekou, R. et al. (1999). Detection of plasmodium falciparum antigen Histidine- Rich Protein-2 in blood of pregnant women, Implication of diagnosing placental malaria. Journal of Clinical Microbiology, 37, 2992-2996. https://doi.org/10.1128/JCM.37.9.2992-2996.1999

Mfuh, K. O., Achonduh-Atijegbe, O. A., Bekindaka, O. N., Esemu, L. F., Mbakop, C. D., Gandhi, K., et al. (2019). A comparison of thick-film microscopy, rapid diagnostic test, and polymerase chain reaction for accurate diagnosis of Plasmodium falciparum malaria. Malaria Journal, $18,73$. https://doi.org/10.1186/s12936-019-2711-4

Mockenhaupt, F. P., Bedu-Addo, G., von Gaertner, C., Boye, R., Fricke, K., Hannibal, I. et al. (2006). Detection and clinical manifestation of placental malaria in Southern Ghana. Malar J., 5, 119. https://doi.org/10.1186/1475-2875-5-119

Nwonwu, E. U., Ibekwe, P. C., Ugwu, J. I., Obarezi, H. C., \& Nwagbara, O. C. (2009).Prevalence of malaria parasitaemia and malaria related anaemia amongst pregnant women in Abakaliki, South East Nigeria. Nigeria Journal of Clinical Practice, 12(2), 182-6.

Odikamnoro, O., Iganga, A., Ozowara, N. L., \& Okoh, N. (2014). Prevalence of malaria among pregnant mothers and possible relationship to parity in Abakaliki, Southeast Nigeria. European Journal of Experimental Biology, 4(4), 15-19.

Perkins, B. A., Zucker, J. R., \& Otieno, J. (1997). Evaluation of an algorithm for the integrated management of childhood diseases in an area of Kenya with high malaria transmission. Bulletin of the World Health Organisation(WHO), 75, 33-42.

Sule-Odu, A. O., Sotunsa, J. O., Adeiyi, T. O., \& Akadri, A. A. (2016). Malaria in pregnancy in Nigeria: Analysis of characteristics of women attending antenatal care in a tertiary facility. Nigerian Medical Practitioner, 69(6), 89-92.

Tan, T. M. C., Nelson, J. S., Ng, H. C., Ting, R. C. Y., \& Kara, U. A. K. (1997). Direct PCR amplification and sequence analysis of extrachromosomal Plasmodium DNA from dried blood spots. Acta Trop., 68, 105-114. https://doi.org/10.1016/S0001-706X(97)00080-6

WHO. (1988).Malaria diagnosis: Memorandum from a WHO meeting. Bulletin of the WHO, 66, 575-594.

WHO. (2009). Malaria microscopy quality assurance manual, version 1.

WHO. (2012).Malaria Rapid Diagnostic Test Performance;Results of WHO product testing of malaria RDTs: Round 4. Retrieved from www.who.int/malaria/publications/atoz/9789241502566/en/index.html. 
WHO. (2015). World Malaria Report. [Last accessed on 7th January, 2017]. Retrieved from http://www.who.int/malaria/publications/world-malaria-report-2015/wmr2015-profiles.pdf

WHO. (2018). Global Malaria Programme Information note on recommended selection criteria for procurement of malaria rapid diagnostic tests.Retrieved from www.who.int/malaria/diagnosis_treatment/diagnosis/RDT_selection_criteria.pdf.

WHO-WPRO/FIND/UNDP/World Bank/WHO-TDR/FIND/GMP. (2010). Methods manual for laboratory quality control testing of malaria rapid diagnostic tests, version six. WHO Regional Office for the Western Pacific (WPRO), FIND, United Nations Development Programme (UNDP)/World Bank/WHO Special Programme for Research \&Training in Tropical Diseases (TDR), WHO Global Malaria Programme (GMP). Geneva: World Health Organization $.2010 \mathrm{~b}$ (http://www.wpro.who.int/malaria/NR/rdonlyres/461C306D-D720-43CA-A476-1A250EC3C26A/0/rdt_lab orato-ry_qc_testing_meth_man_v6.pdf, accessed 3rd June, 2016)

\section{Copyrights}

Copyright for this article is retained by the author(s), with first publication rights granted to the journal.

This is an open-access article distributed under the terms and conditions of the Creative Commons Attribution license (http://creativecommons.org/licenses/by/4.0/). 